\title{
A case series: xiphoidectomy for xiphodynia, a rare thoracic wall disorder
}

\author{
Maikel J. A. M. Bakens ${ }^{\prime \#}$, Paul C. M. Andel ${ }^{1 \#}$, Jean H. T. Daemen ${ }^{1,2} \wedge$, Karel W. E. Hulsewé ${ }^{1}$, \\ Yvonne L. J. Vissers ${ }^{1}$, Erik R. de $\operatorname{Loos}^{1}$ \\ ${ }^{1}$ Division of General Thoracic Surgery, Department of Surgery, Zuyderland Medical Centre, Heerlen, The Netherlands; ${ }^{2}$ Faculty of Health, \\ Medicine and Life Sciences (FHML), School for Oncology and Developmental Biology (GROW), Maastricht, The Netherlands \\ Contributions: (I) Conception and design: MJAM Bakens, PCM Andel, ER de Loos; (II) Administrative support: JHT Daemen, YLJ Vissers, KWE \\ Hulsewé; (III) Provision of study materials or patients: MJAM Bakens, PCM Andel; (IV) Collection and assembly of data: MJAM Bakens, PCM \\ Andel, JHT Daemen; (V) Data analysis and interpretation: MJAM Bakens, PCM Andel, JHT Daemen, YLJ Vissers, KWE Hulsewé, ER de Loos; (VI) \\ Manuscript writing: All authors; (VII) Final approval of manuscript: All authors. \\ \#These authors contributed equally to this work and shared first co-authorship. \\ Correspondence to: Erik R. de Loos. Department of Surgery, Division of General Thoracic Surgery, Zuyderland Medical Centre, Henri Dunantstraat 5 , \\ 6419 PC Heerlen, The Netherlands. Email: e.deloos@zuyderland.nl.
}

Background: Xiphodynia is a rare musculoskeletal disorder characterized by pain at the lower anterior chest or epigastric region. Treatment options include oral analgesics, local injection with analgesic or laser therapy. Yet, these often provide only short-term symptom relieve. A definite reduction in pain sensation might be achieved by performing a xiphoidectomy, though studies on its safety and efficacy are scarce. In the current single-centre study the outcomes of xiphoidectomy for xiphodynia are retrospectively assessed.

Methods: All patients undergoing xiphoidectomy for xiphodynia between April 2013 and February 2020 at Zuyderland Medical Centre, Heerlen, the Netherlands, were included in this case series. Pain scores using the Numeric Rating Scale were assessed preoperatively and postoperatively and submitted to the Wilcoxon signed rank test. A clinically significant improvement was defined as a 2-point decrease in Numeric Rating Scale score. In addition, surgical outcomes, including complications and duration of surgery were extracted from the patient records.

Results: A total of 19 patients underwent xiphoidectomy for xiphodynia. The follow-up ranged from 1 to 83 months. Seventeen out of 19 patients showed a clinically relevant improvement in Numeric Rating Scale pain scores where the overall pain scores also revealed a statistically significant decrease from 8 (interquartile range, 7-8) to 0 (interquartile range, $0-0 ; \mathrm{P}<0.001$ ) after surgery. Median procedure time was 29 minutes (interquartile range, 24-38 minutes) and no postoperative complications occurred.

Conclusions: Xiphoidectomy for xiphodynia is a safe and effective surgical procedure with good outcomes on pain relief. Though, future comparative studies are urged to elucidate its value among other treatment options.

Keywords: Xiphoidectomy; xiphodynia; thoracic wall; thoracic surgery

Submitted Nov 27, 2020. Accepted for publication Feb 26, 2021.

doi: $10.21037 /$ jtd-20-3396

View this article at: http://dx.doi.org/10.21037/jtd-20-3396

^, ORCID: 0000-0002-4878-3951. 


\section{Introduction}

Xiphodynia is a rare musculoskeletal disorder that was first described in 1955 (1). The disorder is characterized by low anterior chest pain or pain in the epigastric region that can irradiate to the throat, back or shoulders, mimicking a series of life-threatening diseases (e.g., myocardial infarction and pulmonary embolism) (2-6). Xiphodynia is therefore usually considered per exclusionem $(7,8)$. Diagnosis mainly relies on physical examination and reproduction of pain symptoms upon manual compression (6), while a crosssectional imaging derived xiphisternal angle less than 160 degrees and bifurcated- or elongated xiphoid processes strengthens the diagnosis $(7,9)$. The exact aetiology is unknown. However, potential causes include inflammation of the xiphoid process due to mechanical injury $(5,6,10)$, anterior protrusion due to excessive weight loss (9) and prior surgery affecting the upper abdominal or lower thoracic region (e.g., upper median laparotomy or median sternotomy) $(2,7,11)$. Numerous non-invasive pain therapies have been recommended, including oral analgesics, local infiltration with analgesic and anti-inflammatory drugs, low level laser therapy and topical anti-inflammatory gel. Yet, they often only provide short-term improvement $(3,6,9,11)$. Surgical resection of the affected xiphoid has been proposed as a definitive treatment, however, studies describing its safety and effectiveness are scarce. The current largest series is published by Dorn and colleagues, who described significant pain relief in 8 out of 10 patients following surgery (7); urging the need for more evidence. At our high-volume tertiary referral centre for chest wall surgery, xiphoidectomy for xiphodynia has been performed since 2013. In this retrospective study we present our singleinstitution experience and assess the safety and efficacy of xiphoidectomy for xiphodynia. We present the following article in accordance with the AME Case Series reporting checklist (available at http://dx.doi.org/10.21037/jtd-203396).

\section{Methods}

\section{Design and setting}

A retrospective, single-centre case-series was conducted of all patients who underwent a xiphoidectomy for xiphodynia between April 2013 and February 2020 at Zuyderland Medical Centre, Heerlen, the Netherlands. The study was conducted in accordance with the Declaration of Helsinki (as revised in 2013). This study was approved by the local medical ethical board (ID: METCZ20200066, approval date: April $9^{\text {th }}$, 2020). Informed consent was retrieved from all patients. The report was written in compliance with the case report (CARE) guidelines (12).

\section{Participants}

All patients who underwent xiphoidectomy for xiphodynia were eligible for inclusion and identified through retrospective review of all thoracic procedures performed between April 2013 and February 2020. No exclusion criteria were drafted. Eligible participants were identified by two independent researchers ( $\mathrm{MB} \& \mathrm{PA})$.

\section{Surgical technique}

Xiphoidectomy was performed under general anesthesia with a single lumen tube. Two grams of prophylactic cefazolin was administered intravenously. Patients were placed in the supine position and the site of surgical incision was marked (Figure 1). After sterile exposure, a $4 \mathrm{~cm}$ longitudinal midline incision was made over the xiphoid process. After partially opening the linea alba in longitudinal direction, the xiphoid process was dissected from its surrounding tissues (Figure 2) and resected in total (Figure 3). The fascia was re-approximated with absorbable, braided sutures (Vicryl 1-0, Ethicon, Somerville, New Jersey, USA; Figure 4). No mesh repair was performed. The subcutaneous tissue and skin were closed in layers.

\section{Measurements and variables}

Data was retrospectively extracted from the available electronic patient records by a single researcher (MB). Extracted variables encompassed baseline patient characteristics (age, sex, body mass index, comorbidities, symptoms and their duration, prior conservative treatments, xiphisternal angle, xiphoid process length, and preoperative pain score) and procedural characteristics (duration of surgery, postoperative length of hospital stay and complications up to 30 days after surgery). Xiphisternal angle (see Figure 5) and xiphoid process length were measured in all patients who preoperatively received either Computed Tomography (CT) or lateral plain radiographs. All measurements were performed by a single author (PA) and validated for correctness by a second author (MB). 


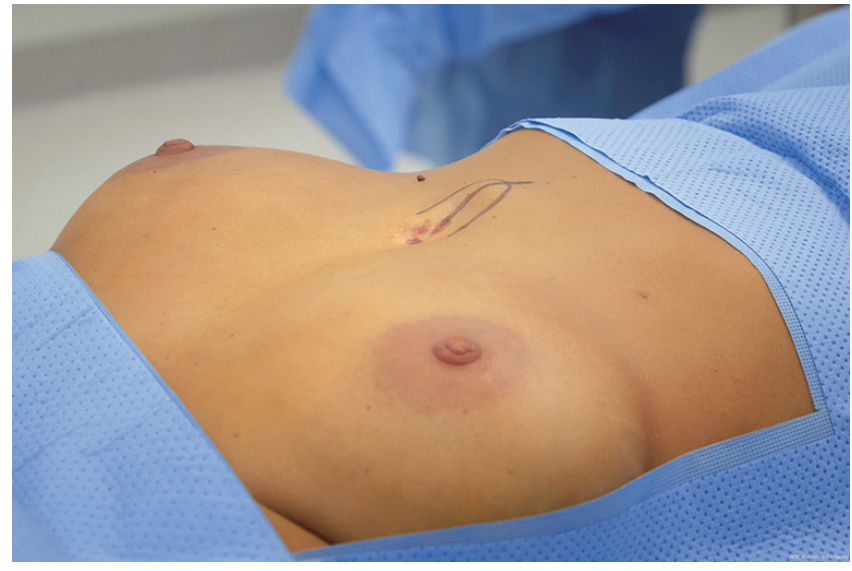

Figure 1 Positioning and skin marking for surgical incision.

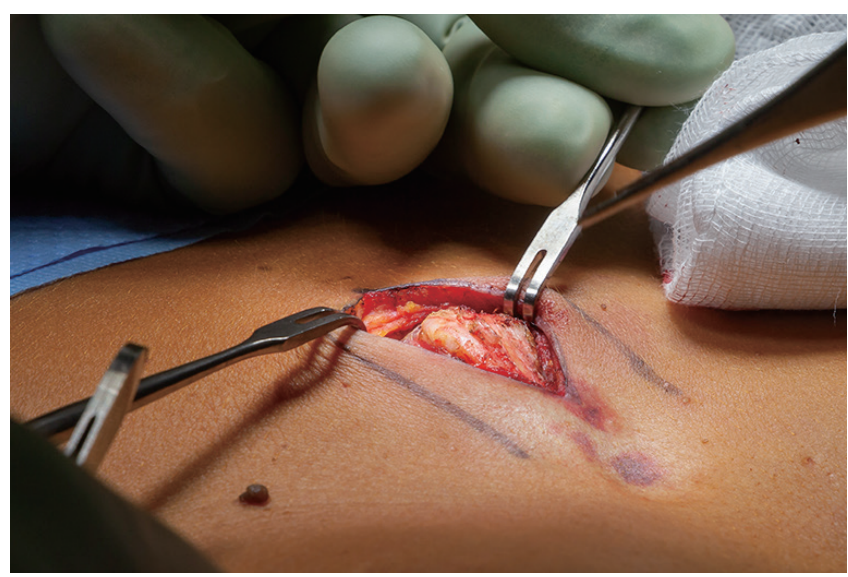

Figure 2 Dissection of the xiphoid process from its surrounding tissue.

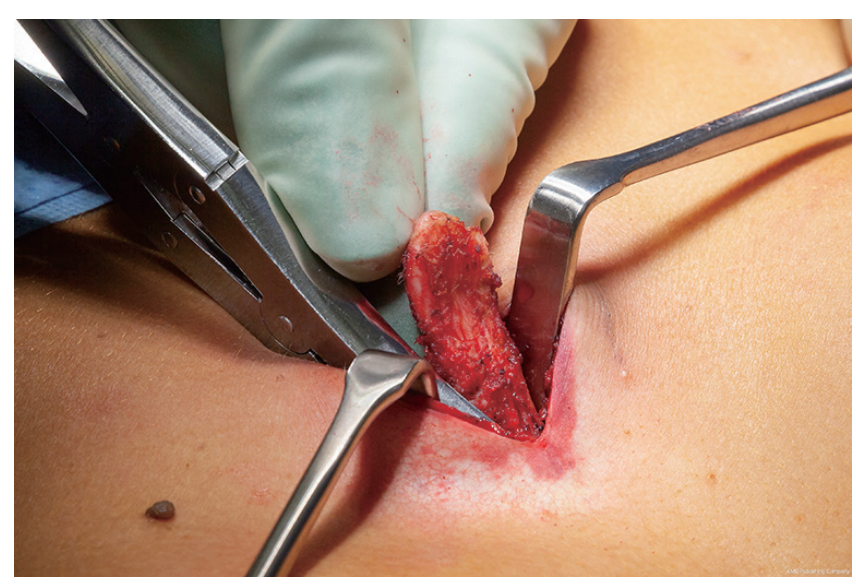

Figure 3 Resection of the xiphoid process.

(c) Journal of Thoracic Disease. All rights reserved.

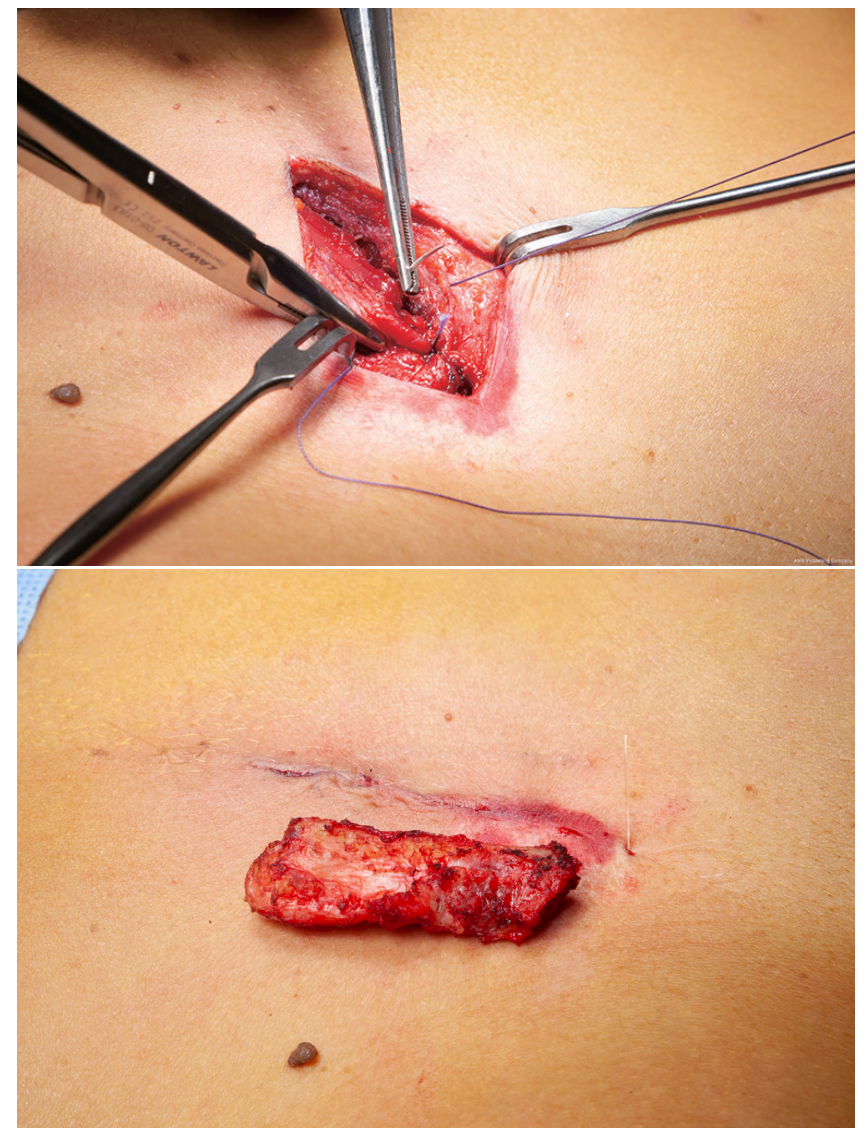

Figure 4 Re-approximation after xiphoidectomy and view after skin closure showing the resected xiphoid process.

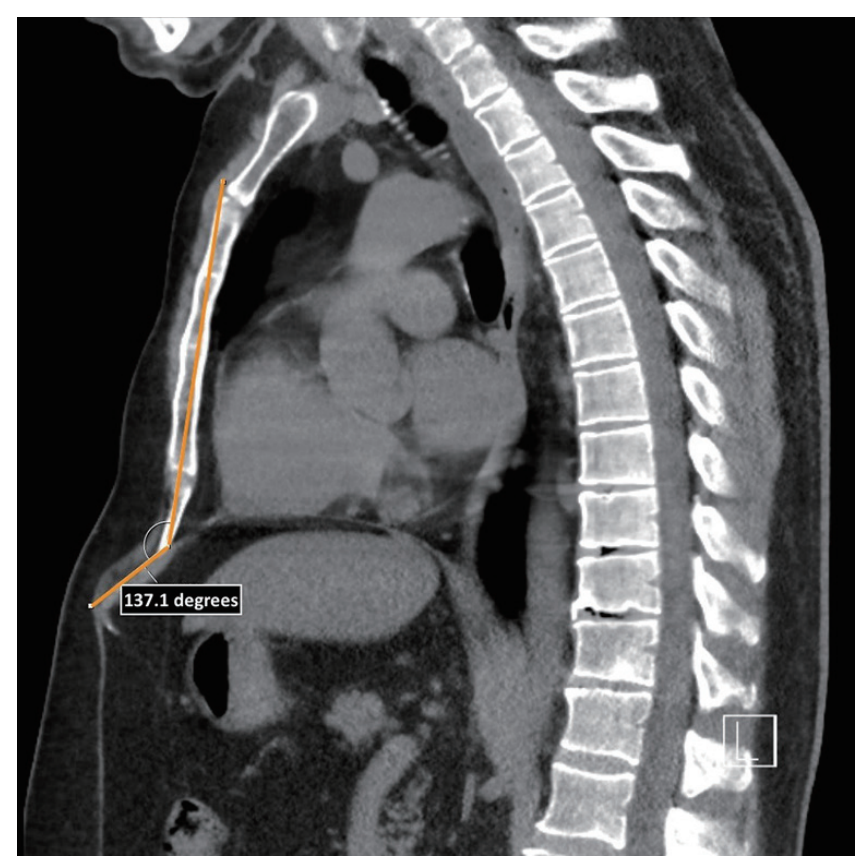

Figure 5 Measurement of the xiphisternal angle. 
Table 1 Patient characteristics

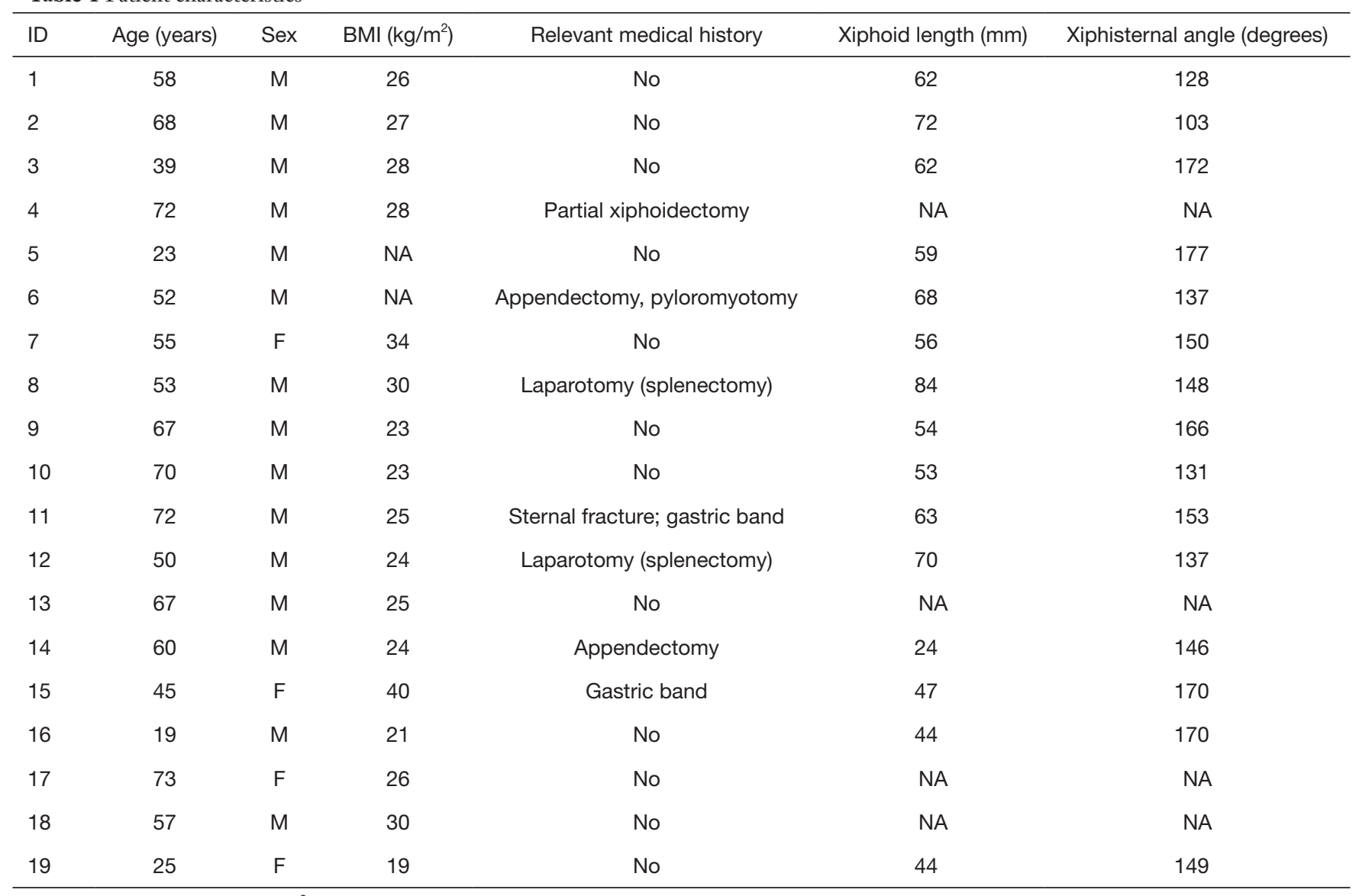

BMI, body mass index; kg/m², kilogram per square meter, mm, millimeter, $\mathrm{M}$, male; NA, not available; $\mathrm{F}$, female.

Pain scores were measured preoperatively at the outpatient clinic using the Numeric Rating Scale (NRS; range, 0 to 10). In addition, postoperative pain scores were gathered by telephone consultation in March 2020. Complications were graded by the Clavien-Dindo Classification (13).

\section{Statistical analysis}

Standard statistical analyses were performed using SPSS statistics (IBM Corp. IBM SPSS statistics for Windows, Version 26.0, Armonk, NY, USA). Categorical variables were depicted as frequency and percentage. Numerical variables were summarized using the median and interquartile range (IQR). Pre- and postoperative NRS scores were submitted to the Wilcoxon signed rank test. A $\mathrm{P}<0.05$ was considered statistically significant. A clinically relevant difference was defined as a 2-point increase or decrease in NRS scores derived from the article from Bird et al. (14). Missing data were reported as such.

\section{Results}

Between April 2013 and February 2020, a total of 19 patients underwent xiphoidectomy for xiphodynia and were included. No patients denied participance or were lost to follow-up. Follow-up ranged from 1 to 83 months. Baseline patient characteristics are depicted in Table 1. Fifteen patients (79\%) were male with a median age of 57 years (IQR: 45-68 years) and a median BMI of $26 \mathrm{~kg} / \mathrm{m}^{2}$ (IQR: $23-29 \mathrm{~kg} / \mathrm{m}^{2} ; \mathrm{n}=17$ ). Six patients previously underwent abdominal surgery, one patient underwent a partial xiphoidectomy and another patient had a history of traumatic sternal fracture.

All patients complained of pain near the xiphoid process or epigastric region which was present for 1 month and up to 35 years. In addition, symptoms were revivable by palpation and other causes were, if suspected, excluded prior to surgery. All patients were treated by conservative therapy prior to consultation with per oral pain therapy varying in 
Table 2 Patient reported outcomes of xiphoidectomy

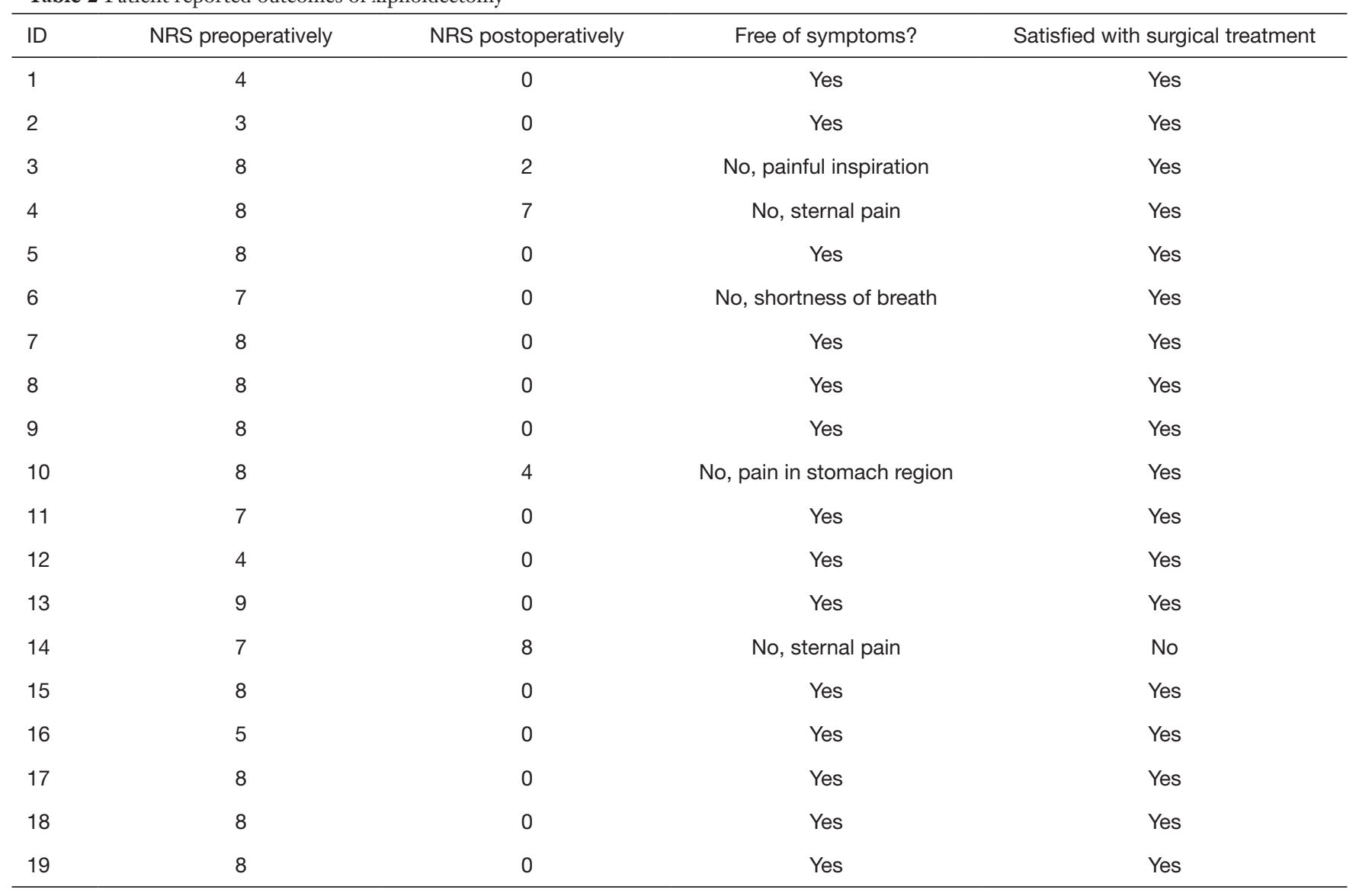

NRS, numeric rating scale.

type (including paracetamol, NSAIDs and opioids) and/or local analgesic injection(s) and duration. No specific drugs for neuropathic pain were provided (e.g., gabapentin). The median xiphisternal angle was 149 degrees (IQR: 137-170 degrees; $\mathrm{n}=15$ ), while the median xiphoid length was $59 \mathrm{~mm}$ (IQR: 47-68 mm; $\mathrm{n}=15$ ).

Median duration of surgery was 29 minutes (IQR: 24-38 minutes; $\mathrm{n}=17$ ). No per- and postoperative complications occurred. The median length of postoperative hospital stay was 1 day (IQR: 1-1). Median preoperative NRS score was 8 (IQR: 7-8) and was reduced to a median NRS score of 0 (IQR 0-0) postoperatively, providing a clinically relevant improvement in seventeen out of 19 patients (see Table 2 and Figure 6). The Wilcoxon signed rank test demonstrated a statistically significant decrease in NRS score after surgery $(\mathrm{P}<0.001)$.

Fourteen patients $(74 \%)$ were completely free of symptoms after surgery. Of the remaining 5 patients, 4 complained of residual pain and 1 complained of dyspnea, already present preoperatively, most probably caused by a different etiology. Despite residual pain, two out of 4 patients demonstrated a clinically significant decline in NRS score, while one of the remaining two patients underwent a prior partial xiphoidectomy. Eighteen out of 19 patients $(95 \%)$ indicated to choose again for surgical treatment. The patient that would not opt for surgery again was the single patient that underwent the prior partial xiphoidectomy. In addition, all but one patient reported excellent postoperative cosmetic results.

\section{Discussion}

The current study shows that xiphoidectomy is an effective surgical treatment option for xiphodynia. Seventeen out of 19 patients showed a clinically significant decline in NRS score of whom 14 were completely free of symptoms. Despite xiphoidectomy provided excellent results in $74 \%$ of participants, we advocate for careful patient selection and 


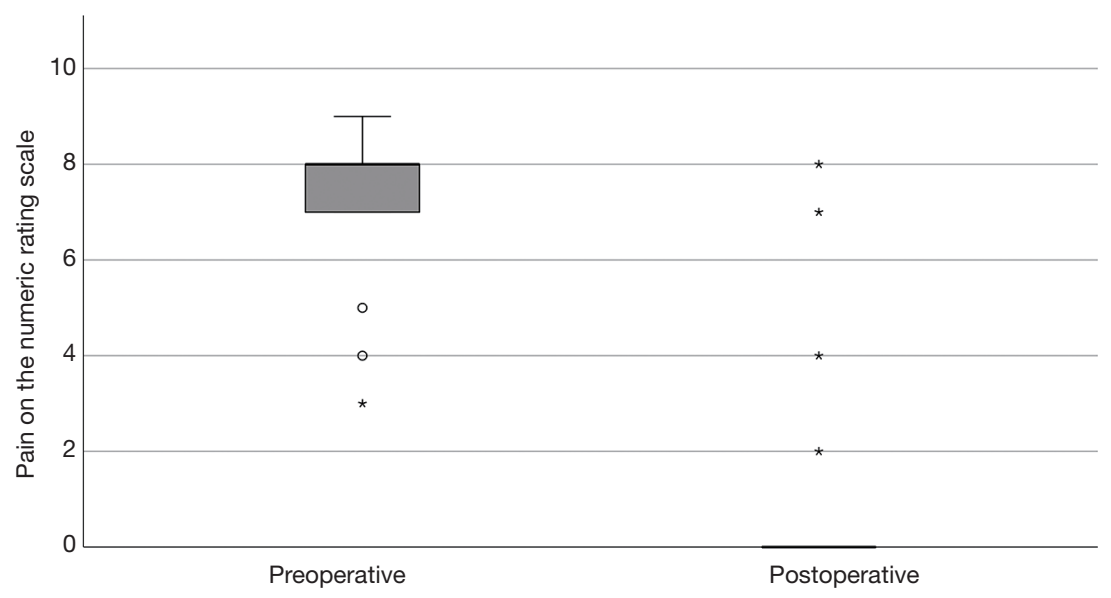

Figure 6 Box plot demonstrating the pre- and postoperative patient reported numeric rating scale score.

work-up before opting for surgery. This should routinely include conservative therapy (e.g., local analgesic injections) and cross-sectional imaging in order to strengthen the diagnosis and optimize outcome. On the contrary, 3 out of 5 patients that were not free of symptoms after surgery received local injections with analgesics prior to xiphoidectomy with good, though only partial response. We therefore also advocate for careful but aggressive exclusion of other causes, such as cardiac, pulmonary and gastrointestinal causes. Xiphoidectomy should in our eyes only be considered if the diagnosis is inevitable and above all not to be executed if there is even the slightest doubt.

Due to being rare, xiphodynia is an underreported and under-recognized disease. Though, Lipkin et al. previously suggested a prevalence of $2 \%$ (1). Although the exact mechanism of pain in xiphodynia remains unclear, disease onset seems to be provoked by factors including xiphoid process elongation and small xiphisternal angels. In addition, luxating factors such as repeated mechanical injury seem to play a role (4). Furthermore, $79 \%$ of patients were male, suggesting that the male sex is a predisposing factor for xiphodynia. This is in accordance with the case series by Dorn et al, in which 11 out of 11 patients were male (7). Conclusively, the male sex might play a role in the pathophysiology of xyphodynia.

Xiphodynia is commonly diagnosed when symptoms can be provoked by manual pressure on the xiphoid process. Cross-sectional imaging can be used to strengthen the diagnosis through assessment of the xiphisternal angle $(<160$ degrees) or by presenting a bifurcated- or elongated xiphoid process, as also stressed by Dorn et al. and Maigne et al. $(7,9)$.
In our series we found a median xiphisternal angle of 149 degrees and xiphoid length of $59 \mathrm{~mm}$. Despite their use for diagnosis, we found no correlation between imaging-based measurements and treatment outcomes.

Most commonly, xiphodynia is primarily treated symptomatically using non-invasive pain therapies including oral analgesics and local infiltration with analgesics and antiinflammatory drugs $(1,3,5,7)$. Unfortunately, conservative treatment does not always provide long-term improvement. Surgical resection of the xiphoid process is a method which has showed to be a promising curative option $(3,7,9,15)$. The current study shows that xiphoidectomy is a safe procedure without complications and adequate pain reduction; up to $74 \%$ of patients were completely free of pain following surgery. The median length of stay was 1 day, providing the ability for xiphoidectomy in an outpatient setting.

Our surgical technique included fascial closure by absorbable sutures. Its rationale was the potential for soft tissue irritation associated with non-absorbable sutures. This is in concordance with the meta-analysis of Hodgson et al., who described reduced pain and wound sinuses using absorbable versus non-absorbable sutures. On the contrary, the use of non-absorbable sutures is hampered by an increased risk for incisional hernia (16). Despite the small sample size, no cases of incisional hernia were encountered in the present series.

The current study is the largest cohort of xiphoidectomies for xiphodynia presented so far. However, there are a number of limitations. The study is a retrospective cohort study, conducted over the past 7 years. Such studies are known to be limited by bias due to missing data, however, because 
the missing data was missing randomly, it was still deemed representative. Since xiphodynia is a rare disease, the included population was small and heterogeneous, limiting scientific value. Yet, this is the largest series to date and thus presenting the best available evidence on disease characteristics and xiphoidectomy as surgical treatment option for xiphodynia. Another limitation is that not all patients received a standardized conservative therapy before surgery.

Despite the NRS was not specifically validated for patients receiving thoracic surgery, it was employed to for patients to rate their pain. The drawback of using such scores is that they are subjective to individual pain experiences. Due to the retrospective study design of the current study, pain scores could not be gathered at specific time intervals after surgery. Furthermore, it was not possible to draw a comparison between the various noninvasive treatment options and surgical intervention. Such a comparative study should be subject of future research to elucidate the role of xiphoidectomy among the different treatment options.

In summary, xiphodynia is a disorder which should be thought of after life threatening diseases and other, more benign disease, are ruled out. Following diagnosis, conservative treatment using oral analgesics and local infiltration with analgesics and anti-inflammatory drugs is often performed as a first treatment which we also advocate. Nevertheless, in many cases this proves to be insufficient for long-term pain relief and surgical treatment may be opted. Xiphoidectomy is, as demonstrated by our series, a safe and effective treatment to reduce and in the majority of cases eliminate pain resulting from xiphodynia with negligible postoperative morbidity.

\section{Conclusions}

Xiphoidectomy for xiphodynia is a safe and effective surgical procedure with good outcomes on pain relief when conservative therapy fails. Though, future comparative studies are urged to elucidate its value among other treatment options.

\section{Acknowledgments}

Funding: None.

\section{Footnote}

Reporting Checklist: The authors have completed the AME
Case Series reporting checklist. Available at http://dx.doi. org/10.21037/jtd-20-3396

Data Sharing Statement: Available at http://dx.doi. org/10.21037/jtd-20-3396

Peer Review File: Available at http://dx.doi.org/10.21037/jtd20-3396

Conflicts of Interest: All authors have completed the ICMJE uniform disclosure form (available at http://dx.doi. org/10.21037/jtd-20-3396). The authors have no conflicts of interest to declare.

Ethical Statement: The authors are accountable for all aspects of the work in ensuring that questions related to the accuracy or integrity of any part of the work are appropriately investigated and resolved. The study was conducted in accordance with the Declaration of Helsinki (as revised in 2013). This study was approved by the local medical ethical board (ID: METCZ20200066, approval date: April 9th, 2020) and individual consent was retrieved from all patients.

Open Access Statement: This is an Open Access article distributed in accordance with the Creative Commons Attribution-NonCommercial-NoDerivs 4.0 International License (CC BY-NC-ND 4.0), which permits the noncommercial replication and distribution of the article with the strict proviso that no changes or edits are made and the original work is properly cited (including links to both the formal publication through the relevant DOI and the license). See: https://creativecommons.org/licenses/by-nc-nd/4.0/.

\section{References}

1. Lipkin M, Fulton LA, Wolfson EA. The syndrome of the hypersensitive xiphoid. N Engl J Med 1955;253:591-7.

2. Eulálio Filho WM. Xiphodynia: a rare cause of epigastric pain. Intern Emerg Med 2018;13:127-8.

3. Hogerzeil DP, Hartholt KA, Vries MR De, et al. Case Report Xiphoidectomy: A Surgical Intervention for an Underdocumented Disorder. Case Rep Surg 2016;2016:9306262.

4. Tanaka Y, Sakata K, Waseda Y, et al. Xiphodynia Mimicking Acute Coronary Syndrome. Intern Med 2015;54:1563-6.

5. Simpson JK, Hawken E. Xiphodynia: A diagnostic 
conundrum. Chiropr Osteopat 2007;15:13.

6. Howell JM. Xiphodynia: a report of three cases. J Emerg Med 1992;10:435-8.

7. Dorn P, Kuhn MA, Schweizer BA, et al. Xiphoidectomy for Intractable Xiphodynia. World J Surg 2018;42:3646-50.

8. Koren W, Shahar A. Xiphodynia Masking Acute Myocardial Infarction: A Diagnostic Cul-de-Sac. Am J Emerg Med 1998;16:177-8.

9. Maigne JY, Vareli M, Rousset P, et al. Xiphodynia and prominence of the xyphoid process. Value of xiphosternal angle measurement: Three case reports. Joint Bone Spine 2010;77:474-6.

10. Yapici Ugurlar O, Ugurlar M, Ozel A, et al. Xiphoid syndrome: an uncommon occupational disorder. Occup Med (Lond) 2014;64:64-6.

11. Enomoto N, Tayama K, Kohno M, et al. Postoperative elongation of the xiphoid process-report of a case. Ann

Cite this article as: Bakens MJAM, Andel PCM, Daemen JHT, Hulsewé KWE, Vissers YLJ, de Loos ER. A case series: xiphoidectomy for xiphodynia, a rare thoracic wall disorder. J Thorac Dis 2021;13(4):2216-2223. doi: 10.21037/jtd-20-3396
Thorac Cardiovasc Surg 2011;17:307-9.

12. Riley DS, Barber MS, Kienle GS, et al. CARE guidelines for case reports: explanation and elaboration document. J Clin Epidemiol 2017;89:218-35.

13. Dindo D, Demartines N, Clavien PA. Classification of surgical complications: A new proposal with evaluation in a cohort of 6336 patients and results of a survey. Ann Surg 2004;240:205-13.

14. Bird SB, Dickson EW. Clinically significant changes in pain along the visual analog scale. Ann Emerg Med 2001;38:639-43.

15. Sano A, Inui M. Xiphoidectomy for xiphoid processinduced pain in a surfer. Asian Cardiovasc Thorac Ann 2015;23:1116-8.

16. Hodgson NC, Malthaner RA, Ostbye T. The search for an ideal method of abdominal fascial closure: a meta-analysis. Ann Surg 2000;231:436-42. 